\title{
An Optimal Control Algorithm Research of Biological Image Quality based on Quantum and Wavelet
}

\author{
Mingjun Wang ${ }^{1}$, Yuan Xiong ${ }^{2}$, Meiliang Wang ${ }^{1}$, Wenyao Zhu ${ }^{1 *}$ and Shuxian \\ Deng $^{3}$ \\ ${ }^{1}$ Engineering and design College, Lishui University, Lishui, China \\ ${ }^{2}$ Zhejiang Vocational College of Commerce, Hangzhou, China \\ ${ }^{3}$ College of Science, Henan Institute of Engineering, Zhengzhou, China \\ *Correspondence Author \\ mingjun_w@163.com
}

\begin{abstract}
This paper introduces an algorithm of wavelet biological image denoising integrated into quantum-inspired method, which is based on the basic principles of quantum mechanics. The quantum-inspired method is applied to estimate the respective probability of wavelet coefficients of the noise and signal of the biological image, then dynamically self-adapt to estimate the semi-soft threshold of wavelet coefficients, so as to protect the signal wavelet coefficients and filter the noise wavelet coefficient. Therefore, the proposed algorithm can restore the noise biological image and protect details of the biological image. Experimental results show that the proposed algorithm can improve the image quality and it is better than other denoising algorithms.
\end{abstract}

Keywords: optimal control, wavelet transform, image denoising, quantum mechanics, biological image

\section{Introduction}

Image is a main medium that human obtains information. The image reflects the objective things of nature and it is an important way to cognize the world. With the development of computer technology, image processing by hand can be completed by computer. In order to make computer automatically process the image, the image must be transformed to the digital form. The digital image in our daily life plays a very important role and it is closely related to our daily life.

In general, the digital image in the process of generating and transmitting can be polluted by a variety of noises. If Signal and Noise Rate (SNR) of the polluted image is lower than a special threshold, then the expression of the image scene context is affected. Meanwhile, these noises might cover some important details in the digital image. The pollution generated from the process of collecting and obtaining can be improved by updating hardware devices, but, the pollution generated in the process of coding and transmitting must apply denoising technology to promote the quality of the image.

In the process of taking plankton photos, due to the influence of other microbes or impurities in the ocean, the following serious noises are likely to exist. Firstly, the plankton is a live body and moves in the water, thus, such relative movement can cause blurring and contour distortion. Secondly, an effect of light scattering and absorption in the seawater makes the image quality lower. Thirdly, changes such as light source intensity, angle and imaging distance can cause the image quality lower. Finally, refection causes uniform illumination, which is generated by a variety of suspended particles and the planktons of different forms, and makes the image edge blur. 


\section{Quantum-Inspired Algorithm}

Quantum mechanics, which is one of the most important scientific achievements in physics field since twenty-first Century [1-2], reveals structure and properties of microscopic particles. Laws of quantum mechanics not only dominate the microscopic world, but also dominate the macroscopic world. The microscopic particles have waveparticle dualism. When the size of particle is from micro to macro, the particle follows the laws from quantum mechanics to classical mechanics. In fact, classical physics, which describes the macroscopic material movement laws, is only an approximation of quantum mechanics. Quantum mechanics can not only explain a series of phenomena of micro world, but also be applied to perfectly explain the macroscopic object movement.

Wave-particle dualism such as superposition principle, quantum entanglement and statistical uncertainty, can reflect the image randomness on some level. Through the exploration of biological evolution mechanisms, such as genetic, neural network, DNA computing and so on, human beings got the inspiration and came up with the quasiphysical idea. This paper uses the basic concept and theory of quantum mechanics, such as coherence, entanglement and superposition of quantum, introduces a refined method which solves the image processing problem by the application of the quantum properties, the combination of quantum mechanics theory and the image processing technology. We not only obtain a better image processing effect, but also introduce a new theoretical tool for image processing technology.

\section{Wavelet Denoising Algorithm}

Wavelet transform in time-frequency domain has very good locality and has the ability of concentration to certain signal as its variable scale feature. If signal energy concentrates on a small part of wavelet coefficient, then the signal coefficient value is greater than the noise coefficient's. After wavelet transform of the noise image, the coefficients of image signal and noise signal show different characteristics. The signal energy concentrates on some bright line, and most of the coefficient value approaches zero. On the contrary, the noise coefficients are distributed evenly on the entire scale space and their values are smaller than the signal ones. The characteristic provides reason for the image denoising of wavelet transform.

The essence of wavelet denoising is to look for the best mapping from the actual signal space to the wavelet function space, then to get the best recover of original signal. In fact, wavelet denoising is a signal filtering problem. In order to preserve the image characteristic after denoising, feature extraction and low-pass filter function are integrated by wavelet denoising. To seek the process to get rid of the noise based on wavelet transform, which is to seek the best treatment method of wavelet coefficients. According to different processing methods of wavelet coefficients, denoising methods can be divided into three categories: module maximum detection method, wavelet coefficient correlation denoising method, and threshold denoising method.

\subsection{Module Maximum Detection Method [1]}

The signal singularity refers to the signal with discontinuous or a derivative discontinuity in somewhere. Obviously, infinite differentiable function is smooth and with no singularity. The singular point is the signal abrupt change point which contains important features of the signal. From the viewpoint of mathematics, the signal singularity can be measured by Lipschitz index or singular index.

In 1992, S. Mallat combined Lipschitz exponent with local maxima of coefficient module behind wavelet transform, then applied the attenuation rate of different scales to measure the local singularity of the signal. Specifically, they used the modulus maxima of wavelet transform of the useful signal and noise, which shows different singularity in 
multi-scale analysis, and weeded out modulus maxima points generated by noise and remain modulus maxima points generated by signal. Lastly, they applied the rest modulus maxima to estimate the wavelet coefficients, then utilized the wavelet coefficient estimated to recover the signal again.

By using module maximum detection method denoising effect is less dependent on the noise itself and the method is stable performance. Don't need to know more transcendental value of the noise. Especially it has the obvious denoising effect to the low SNR signal. Experiments show that, when the image contains white noise and more singular point, the method can obtain higher SNR and no redundant oscillation. Meanwhile, the method should pay attention to the calculation method of wavelet coefficient estimate and the selection of threshold and scale.

\subsection{Wavelet Coefficient Correlation Denoising Method}

Wavelet domain filtering based on different forms of wavelet transform of the noise and signal in different scales constructs the corresponding removal rules to process the wavelet transform coefficients. The last aim is to reduce or completely eliminate the wavelet transform coefficients corresponding to the noise, simultaneously to retain the wavelet transform coefficients corresponding to the signal. After the wavelet transform the wavelet coefficients in different scales have strong correlation, especially in the near edge of the signal ${ }^{[2]}$ their correlation is more obvious. While, the correlation of the wavelet transform corresponding to the noise is not such obvious between the scales. By the multilevel wavelet transform of the image, the correlation of the wavelet transform coefficients is computed between adjacent scales. The coefficients of signal and noise are distinguished by using the correlation of wavelet coefficient in different scales. By this way, the signal or noise is chosen. Lastly, the signal is recovered from the wavelet coefficient chosen ${ }^{[3,4]}$. The method reserves all the wavelet transform coefficients of lower resolution (a large scale) and these coefficients of points located in the edge (a small scale) with the rest coefficients removed. The coefficients of the noise wavelet transform mainly concentrate on the small scale at all levels, therefore the noise is basically eliminated and the edge information can be better protected.

The correlation denoising method of the wavelet coefficient is simple, which is necessary to complete repeatedly. The correlation coefficient in a point is decided by wavelet coefficients of two adjacent scales. If an error occurs in this process of wavelet decomposition, it can lead not to truly reflect the point correlation. Thus, the point cannot get correct value. In addition, the repeated computing does not finish till the setting threshold. Therefore, these problems such as the efficient calculation of the correlation coefficient and the choosing of the threshold scale are still to be investigated.

\subsection{Threshold-Denoising Method}

The threshold denoising method gets the wavelet coefficients from the wavelet transform to the image. The signal corresponding to the wavelet coefficient contains important information, that data is less and amplitude changes larger. However, the noise wavelet coefficients are the opposite. Therefore, by setting a specific threshold of wavelet coefficients, we can get the estimate value of the wavelet coefficients. Lastly, we can obtain the denoising image through reconstructing the wavelet coefficients.

As the threshold denoising method is simple to perform and is fast to compute, it has been widely used in practice. After dealing with the wavelet coefficients on the threshold, we can reconstruct the wavelet signal with the coefficients. There are two kinds of the threshold methods. One is the hard threshold, which gets the wavelet coefficients of poor continuity and has the oscillation or mutation phenomena to the signal reconstructed. The other is the soft threshold, which obtains the wavelet coefficients of better continuity. But, when the wavelet coefficients are larger, there is certain deviation between the wavelet 
coefficients processed and the actual ones, which may lead to the reconstructed error between them.

When the selected threshold is too large or small, the image detail and edge information cannot be preserved at the same time of denosing. Thus, choosing the appropriate threshold can keep the image detail and edge information. Currently, using range of the threshold in the process of the wavelet transform and reconstruction includes global threshold and local adaptation threshold.

\section{Quantum-Inspired Wavelet Denoising Methods}

\subsection{Models}

In this paper, the image denoising method is for additive noise, if it is a multiplicative noise, logarithmic transformation can be the solution. In the DTCWT wavelet domain the image model can be expressed as:

$$
Y=I+N
$$

where $I=I_{r}+i I_{i}$ and $N=N_{r}+i N_{i}$ respectively denote the complex wavelet coefficients of the original image and noise, $Y=Y_{r}+i Y_{i}$ denotes the complex wavelet coefficients the image noised.

In order to improve the denoising performance of algorithm, we consider the correlation between the scales of the wavelet coefficients in the algorithm proposed. We apply the normalized product of the wavelet coefficients of father and son, and introduce the emergence probability of quantum-inspired signal and noise. The signal wavelet coefficients between the scales, which are related to the nose coefficients, have a strong correlation. In a certain extent, the correlations of the signal coefficients between scalars reflect the signal energy transmission. For the signal coefficients, the bigger the parent coefficient, the bigger the son coefficient ${ }^{[5]} .|\nabla Y(i, j)|$ is the checking operator. Assuming big gradient region corresponding to the strong characteristics, the impact of the noise is not obvious to these regions. Medium gradient region corresponding to the image characteristics avoids reducing the image visibility. Low gradient region corresponding to the noise and equivalent region needs to be smoothed strongly. The image denoising method proposed in this paper, which includes the product of the father and son generation wavelet coefficients and the gradient, is the following:

$$
K_{s \theta}^{i j}=\left|Y_{\theta}(s+1, i, j)\right| \times\left|Y_{\theta}(s, i, j)\right| \times|\nabla Y(i, j)|,
$$

where $\theta$ is the value of six direction, such as $\pm 15^{\circ}, \pm 45^{\circ}, \pm 75^{\circ} ; \nabla Y$ expresses the gradient of the noise image at ${ }^{(i, j)} ; K_{s \theta}^{i j}$ denotes the product of the parent coefficient module $Y_{\theta}(s+1, i, j)$ and the present subband coefficient module on the $\theta$ subband image of the scale $S$ direction. Among wavelet high frequency subband of multi-scale, the wavelet coefficients are essentially a sum of the signal and noise of the image. From these we naturally associate the superposition principle in quantum mechanics. Thus, according to the superposition principle ${ }^{[6]}, K_{s \theta}^{i j}$ can be seen as the superposition of the noise and signal:

$$
\left|K_{s \theta}^{i j}>=a\right| 0>+b \mid 1>,
$$

where $a$ and $b$ denote respectively probability amplitude of the quantum ground state $\mid 0>$ and $\left.|1>;| a\right|^{2}$ and $|b|^{2}$ express respectively measurement probability of the noise state $\mid 0>$ and the signal state $\mid 1>$. Here $|a|^{2}+|b|^{2}=1$. In the high 
frequency subband, the signal is relative with the edge and detail of the image. Supposing that $N K_{s \theta}^{i j}$ is $K_{s \theta}^{i j}$ normalized, and $N K_{s \theta}^{i j} \in[0,1]$. In some extent, $N K_{s \theta}^{i j}$ reflects the emergence probability of the high frequency signal. The bigger the value of $N K_{s \theta}^{i j}$, the bigger the energy. The relationship, which the coefficients of parent and son generation correspond to the same location, has bigger probability to appear the high frequency signal and the image detail. On the contrary, there is bigger possibility appearing the probability of the noise here. In the proposed algorithm the definition of $\left|K_{s \theta}^{i j}\right\rangle$ is the following:

$$
\left|K_{s \theta}^{i j}>=\cos \left(N K_{s \theta}^{i j} \times \pi / 2\right)\right| 0>+\sin \left(N K_{s \theta}^{i j} \times \pi / 2\right) \mid 1>.
$$

Where $\cos \left(N K_{s \theta}^{i j} \times \pi / 2\right)$ and $\sin \left(N K_{s \theta}^{i j} \times \pi / 2\right)$ respectively denotes the occurrence probability of the signal and noise of quantum derivation of the wavelet coefficients at $(i, j)$ in the subband image of the $\theta$ direction of $s$ scale.

This paper introduces into the emergence probability of quantum derivative signal and noise in the high frequency subband, which helps to smooth the image and protect the image details. In our denoising method proposed, the correlation between the scales of the wavelet coefficients is considered, the image details through nonlinear quantum derivative probability is strengthened, the noise is weakened, the robustness is enhanced and the computing complexity is lowered. So, our method has better performance of image denoising.

Dohono and others put forward the concept of the wavelet threshold denoising on the basis of the wavelet transform ${ }^{[9]}$. In the actual signal processing, the wavelet denoising method of hard and soft threshold has been widely used and also achieved good effect. For the noise signal $Y$ of the length $N$, assuming $N=2^{J}$, applying the fast algorithm of orthogonal wavelet transform to get the scale coefficients $\left\{v_{L, k}, k=1,2, \cdots, 2^{L}\right\}$ under the lower resolution $L \quad(0 \leq L<J)$ and the wavelet coefficients $\left\{w_{j, k}, j=L, L+1, \cdots J-1 ; k=1,2, \cdots, 2^{j}\right\}$ under all resolution. Here there are $N$ wavelet transform coefficients.

The denoising function of hard threshold is as follows:

$$
\tilde{w}_{j, k}=\left\{\begin{array}{l}
w_{j, k},\left|w_{j, k}\right|>\xi \\
0,\left|w_{j, k}\right| \leq \xi
\end{array} .\right.
$$

The denoising function of soft threshold is as follows:

$$
\tilde{w}_{j, k}=\left\{\begin{array}{l}
w_{j, k}-\xi, w_{j, k}>\xi \\
0,\left|w_{j, k}\right| \leq \xi \\
w_{j, k}+\xi, w_{j, k}<-\xi
\end{array},\right.
$$

where $\xi=\sigma \sqrt{2 \log _{2} N}$ is the global threshold and $\sigma$ is the standard deviation of the noise signal.

From the denoising threshold function, we can see that the hard threshold function can preserve the local characteristics of the signal edge very well and the denoising effect is better to the mutation signal. Smooth effect of the soft threshold function is more than the hard threshold, but it is easy to cause the distortion phenomenon of the signal edge. In order to combine their advantages, we adopt an adaptive semi-soft threshold denoising method, through introducing the noise probability of quantum derivation and model 
parameter $\xi$ to adaptive estimate $\hat{\xi}$. As shown in figure 1 , drawing several different curves corresponding to the values of $\sigma$. The expression is as follows:

$$
\hat{\xi}=\xi\left(1+\cos ^{2}\left(N K_{s \theta}^{i j} \times \pi / 2\right)\right)
$$

The function expression of adaptive semi-soft threshold is as follows:

$$
\tilde{w}_{j, k}=\left\{\begin{array}{l}
w_{j, k}-\hat{\xi}+\frac{\hat{\xi}}{2 j+1}, w_{j, k}>\hat{\xi} \\
\frac{1}{(2 j+1) \hat{\xi}^{2 j}} w_{j, k}^{2 j+1},\left|w_{j, k}\right| \leq \hat{\xi} . \\
w_{j, k}+\hat{\xi}-\frac{\hat{\xi}}{2 j+1}, w_{j, k}<-\hat{\xi}
\end{array}\right.
$$

From (6) we can see that, the semi-soft threshold function exists a smooth transitional region between the signal and noise, which accords with the characteristic of natural images. Figure 2 shows the graphical representation of semi-soft threshold method. Meanwhile, the bigger the noise probability of quantum derivation corresponding to the position (its value tends to 1), the bigger the semi-soft threshold adaptively in (6), so that filtering out the noise very well. On the other hand, the less the noise probability of quantum derivation (its value tends to 0 ), the more the probability of quantum derivation signal corresponding to the wavelet coefficients. At this point, the semi-soft threshold of Eq. (6) is adaptively decreased, which preserves the image details such as edge, texture. The results of our experiments show that the adaptive semi-soft threshold method combines the advantage of the hard and soft threshold and the de-noising effect is obvious.

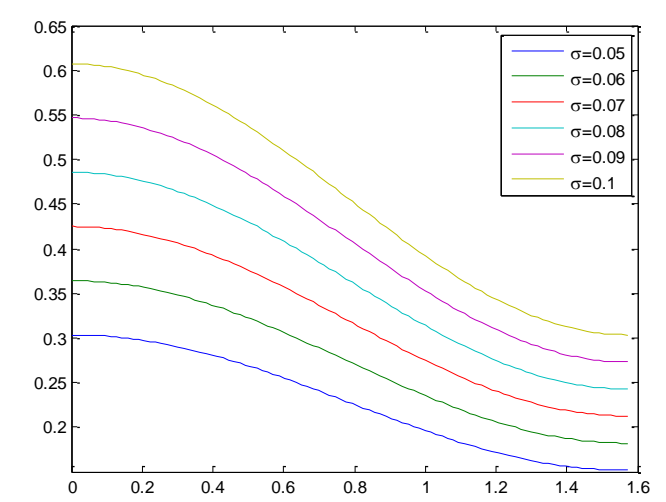

Figure 1. $\hat{\xi}$ Adaptive Estimate

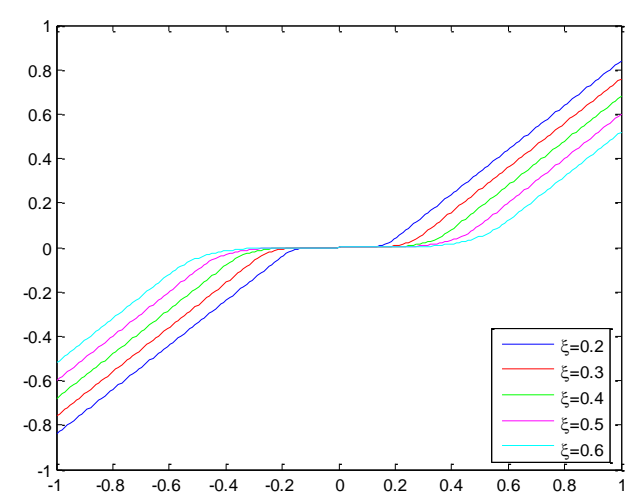

Figure 2. Semi-Soft Threshold

\subsection{Algorithm}

On the biological image denoising problem, the correlation between the scales of the wavelet coefficients is considered enough. According to the father and son generation wavelet coefficients of the high frequency subband and the product of the noise image gradient, the probability of quantum derivation is introduced. Through the setting of adaptive semi-soft threshold parameter and modifying the wavelet coefficients adaptively, this method can improve the de-noising performance of the algorithm and preserve the image details. The steps of the image de-noising algorithm are as follows: 
1) Using DTCWT to transform the noise image Y.

2) According to the noise probability of quantum derivation in (4), computing the adaptive parameter $\hat{\lambda}$.

3) Modifying the wavelet coefficients by the semi-soft threshold method adaptively.

4) Reconstructing the signal through DTCWT inverse transformation.

In this paper, the biological image de-noising method proposed enough considers the correlation between the scales of the wavelet coefficients. So, the image denoising performance is improved.

\section{Experiments and Analysis}

In this paper, we apply these indexes of peak signal noise ratio (PSNR) and edge keeping degree (EKD) to evaluate the de-noising performance.

1) PSNR is defined as the following:

$$
P S N R=10 \log \frac{255^{2}}{M S E},
$$

where MSE is defined as follows:

$$
M S E=\frac{1}{m \times n} \sum_{i=1}^{m} \sum_{j=1}^{m}(x(i, j)-y(i, j))^{2} .
$$

2) EKD is defined as the following:

$$
E K D=\frac{\Gamma(\Delta F-\bar{\Delta} F, \Delta F-\bar{\Delta} F)}{\sqrt{\Gamma(\Delta F-\bar{\Delta} F, \Delta F-\bar{\Delta} F) \Gamma \Gamma(\Delta F-\bar{\Delta} F, \Delta F-\bar{\Delta} F)}},
$$

where

$$
\Gamma\left(t_{1}, t_{2}\right)=\sum_{i=1}^{m} \sum_{j=1}^{m} t_{1}(i, j) \times t_{2}(i, j)
$$

Here, $\Delta F$ and $\Delta F$ respectively denote the results of high-pass filtering of $F$ and $F$ through $3 \times 3$ standard Laplacian operator. EKD is the evaluation index to measure the image edge keeping ability. EKD is closed to 1, which shows the effect of the image edge keeping is better.

In order to test the performance of the proposed de-noising algorithm, we apply different de-noising algorithm to process these plankton images such as Copepoda and Daphnia which add Gaussian white noise. We use the comparison methods such as Mean, Wiener ${ }^{[10]}, \mathrm{TV}^{[13]}$, soft and hard threshold ${ }^{[9]}$. From Table 1, the value of PSNR is bigger than other algorithms under the same $\sigma$. From Table 2, EKD is more than other algorithms under the same $\sigma$. Through these datum of Table 1 and Table 2, we get to know that the proposed algorithm has good performance in terms of preserving the image details. Figure 3 and Figure 4 are Copepoda plankton, and Figure 5 and Figure 6 are Daphnia plankton. Due to the limitation of space, this paper only shows these de-noising images of $\sigma=0.05$ Gaussian white noise.

Our proposed algorithm introduces quantum superposition, entanglement and statistical uncertainty in the microscopic particles of quantum mechanics, and truly reflects the image randomness in a certain extent, thus, this algorithm is superior to other algorithms in terms of preserving the details of the biological image. 
Table 1. PSNR (Unit: Db)

\begin{tabular}{|c|c|c|c|c|c|c|}
\hline$\sigma$ & Mean & Wiener & TV & Soft & Hard & $\begin{array}{l}\text { Propose } \\
\text { d }\end{array}$ \\
\hline 05 & 15.3385 & 15.6554 & $\begin{array}{l}15.96 \\
91\end{array}$ & $\begin{array}{l}17.16 \\
64\end{array}$ & 17.2897 & 17.8391 \\
\hline 06 & 15.1834 & 14.7246 & $\begin{array}{l}14.78 \\
02\end{array}$ & $\begin{array}{l}16.99 \\
88\end{array}$ & 17.0021 & 17.5671 \\
\hline 07 & 14.8036 & 13.9363 & $\begin{array}{l}13.68 \\
25\end{array}$ & $\begin{array}{l}16.41 \\
62\end{array}$ & 16.5891 & 16.9761 \\
\hline 08 & 13.8387 & 13.6267 & $\begin{array}{l}12.89 \\
85\end{array}$ & $\begin{array}{l}15.96 \\
23\end{array}$ & 16.0823 & 16.5467 \\
\hline 09. & 13.2890 & 12.9503 & $\begin{array}{l}11.83 \\
28\end{array}$ & $\begin{array}{l}15.18 \\
14\end{array}$ & 15.7893 & 15.9892 \\
\hline 10. & 12.6965 & 12.4747 & $\begin{array}{l}11.32 \\
85\end{array}$ & $\begin{array}{l}14.48 \\
79\end{array}$ & 15.1265 & 15.5933 \\
\hline
\end{tabular}

Table 2. EKD

\begin{tabular}{cclllll}
\hline$\sigma$ & $\begin{array}{c}\text { Me } \\
\text { an }\end{array}$ & $\begin{array}{l}\text { Wien } \\
\text { er }\end{array}$ & TV & Soft & Hard & Proposed \\
\hline $\mathbf{0 . 0}$ & 0.6 & 0.617 & 0.8287 & 0.8290 & 0.836 & 0.8972 \\
$\mathbf{5}$ & 328 & 8 & & & 0 & \\
$\mathbf{0 . 0}$ & 0.6 & 0.589 & 0.7793 & 0.8043 & 0.810 & 0.8573 \\
$\mathbf{6}$ & 067 & 7 & & & 2 & \\
$\mathbf{0 . 0}$ & 0.5 & 0.533 & 0.7294 & 0.7736 & 0.781 & 0.8057 \\
$\mathbf{7}$ & 283 & 8 & & & 2 & \\
$\mathbf{0 . 0}$ & 0.5 & 0.494 & 0.6624 & 0.7125 & 0.718 & 0.7746 \\
$\mathbf{8}$ & 020 & 1 & & & 8 & \\
$\mathbf{0 . 0}$ & 0.4 & 0.486 & 0.6116 & 0.6949 & 0.709 & 0.7284 \\
$\mathbf{9}$ & 927 & 7 & & & 8 & \\
$\mathbf{0 . 1}$ & 0.4 & 0.441 & 0.5358 & 0.6647 & 0.688 & 0.7074 \\
$\mathbf{0}$ & 623 & 9 & & & 3 & \\
\hline
\end{tabular}

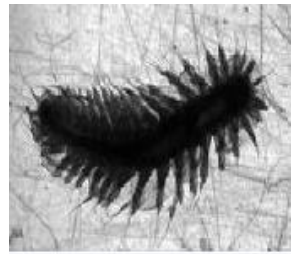

(a) Original Image

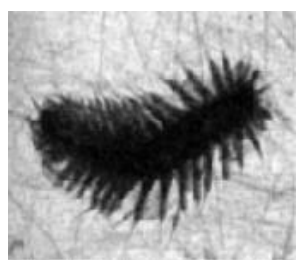

(e) TV

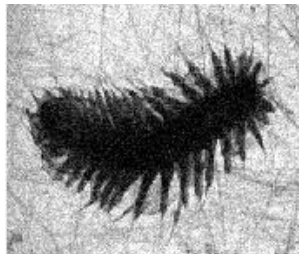

(b) Noise Image $(\sigma=0.05)$

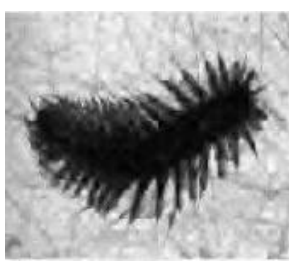

(f) Soft Threshold

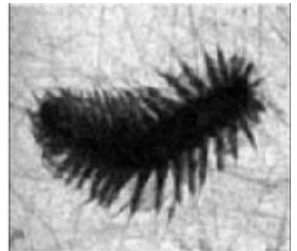

(c) Mean

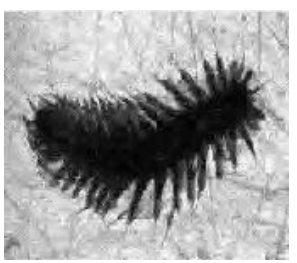

(g) Hard Threshold

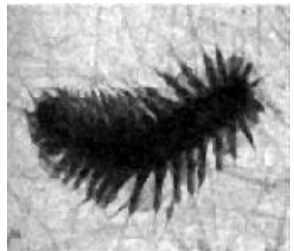

(d) Wiener

Figure 3. Copepoda 1

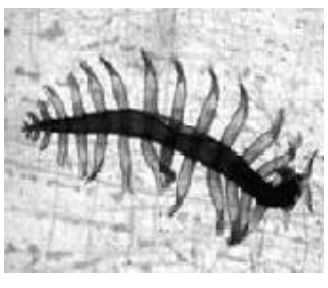

(a) Original Image

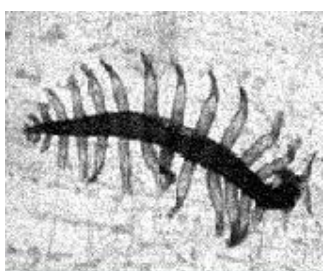

(b) Noise Image

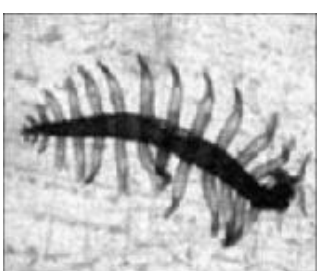

(c) Mean

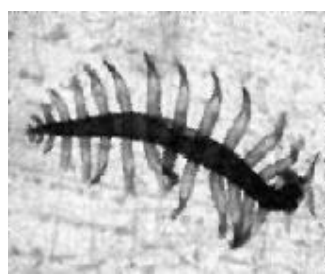

(d) Wiener

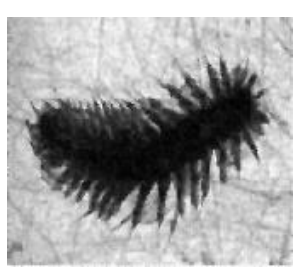

(h) proposed 


\section{$(\sigma=0.05)$}

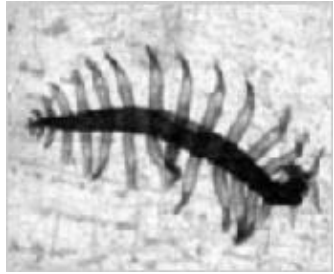

(e) TV

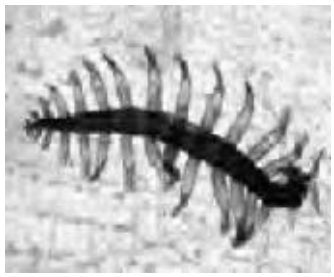

(f) Soft Threshold

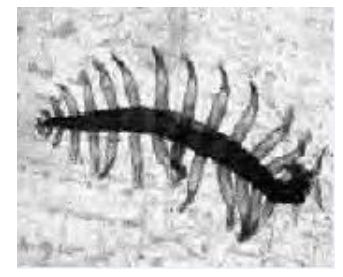

(g) Hard Threshold

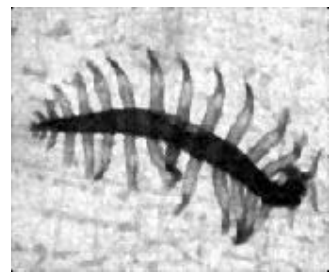

(h) proposed

Figure 4. Copepoda 2

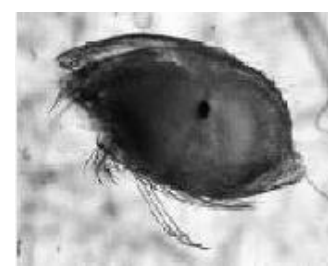

(a) Original Image

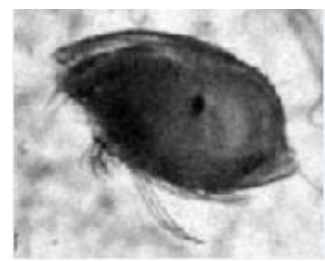

(e) TV

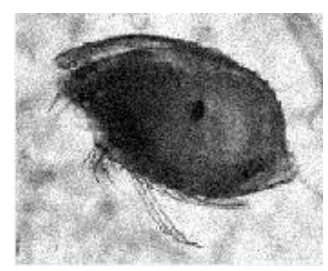

(b) Noise Image $(\sigma=0.05)$

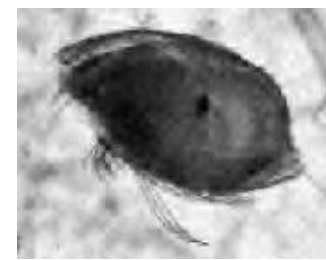

(f) Soft Threshold

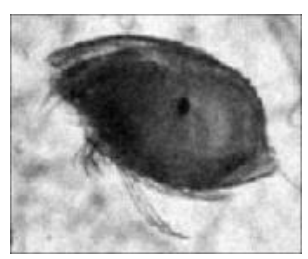

(c) Mean

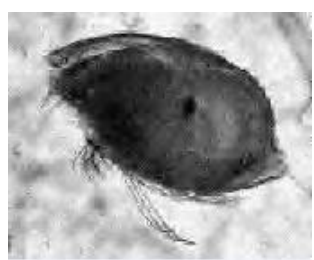

(g) Hard Threshold

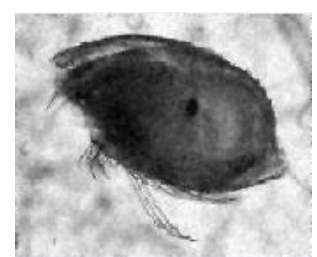

(d) Wiener

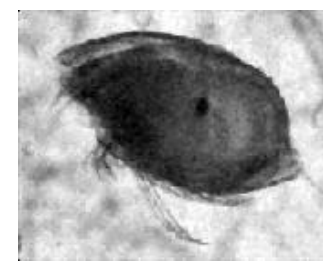

(h) proposed

Figure 5. Daphnia 1

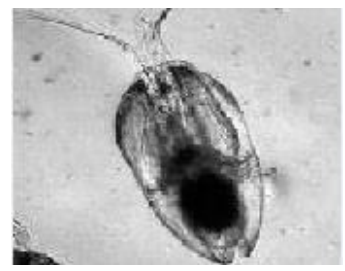

(a) Original Image

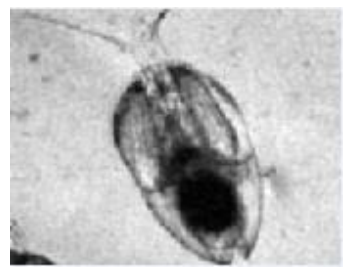

(e) TV

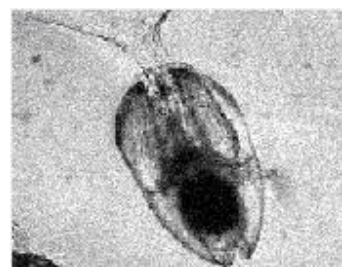

(b) Noise Image $(\sigma=0.05)$

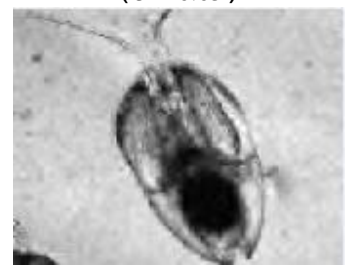

(f) Soft Threshold

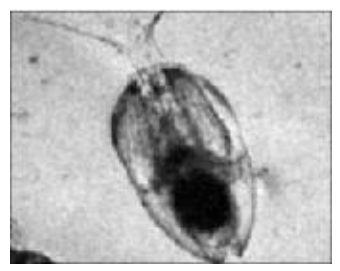

(c) Mean

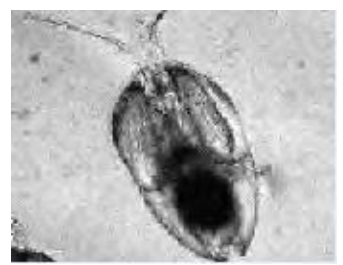

(g) Hard Threshold

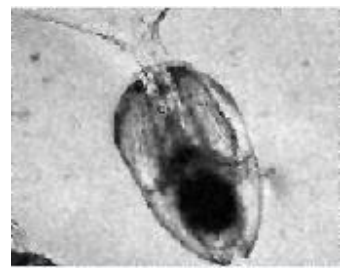

(d) Wiener

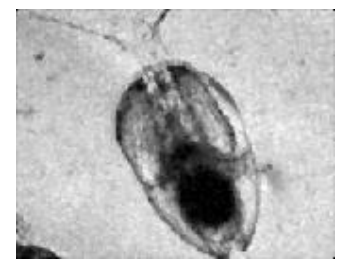

(h) proposed

Figure 6. Daphnia 2 


\section{Conclusions}

For the wavelet image de-noising, the hard threshold de-noising method can well retain local signal characteristics such as edge, but the wavelet coefficients obtained are poor continuity. Meanwhile, using the soft threshold de-noising method can get the rich continuity of the wavelet coefficients. However, it reduces the precision of the reconstructed signal and results in the blurred edges. We adopt the semi-soft threshold which can combine the advantage of the soft and hard threshold. We introduce quantum superposition, entanglement and statistical uncertainty in the microscopic particles of quantum mechanics, and truly reflect the image randomness in a certain extent. Thus, our proposed algorithm can suppress the random noise and keep the image edge and contour features, and is better than the other similar algorithms in terms of the biological image details. In the future research work, we will continue to excavate the basic principle of quantum mechanics and further improve the quality of image de-noising.

\section{Acknowledgements}

The authors would like to thank both anonymous referees for their careful reading and helpful suggestions.

\section{References}

[1] C.S. Burrus, R.A. Gopinathand H.T. Guo, "Introduction to Wavelets and wavelet Transform", Prentic Hall, Upper Saddle River (1998).

[2] I. Daubechies, "The Wavelet Transform: Time-frequency localization and signal analysis", IEEE Traps Theory, 26(9): 961-1005 (2001).

[3] S.M.Temphsne and S.-F. Zhong, "Characrecization of Signal from Multi-scale Edgea [J]", IEEE Trans On Pattern Analysis And Machine Intelligence, 14(7): 710-732 (1992).

[4] M.J.T. Smith and S.L. Eddins, "Analysis/synthesis techniques for subband image coding IEEE Trans", On ASSP, 38: 1446-1456 (1990).

[5] Y. Xu, J. Weaver and D. Healy, "Wavelet transform domain filters: a spatially selective noise filtration technique", IEEE Transactions on Image Processing, 3(6): 747-758 (1994).

[6] Y. Eldarand A. Oppenheim, "Quantum signal processing”, IEEE Signal Processing Magazine, 19(6):1232 (2002).

[7] Y. Sun, T. Lan and X. Fu, "A statistical approach to contour extraction based on quantum mechanics", in: SPIE Medical Image. Orlando, Florida, USA: 7529: 75294N1-75294N9 (2009).

[8] A. Khare, M. Khare and Y. Jeong, "Despeckling of medical ultrasound images using Daubechies complex wavelet transform", Signal Processing, 428-439 (2009).

[9] D.L. Donoho, I.M. Johnstone, "Ideal Spatial Adaptation by Wavelet Shrinkage", Biometrika, 81: 425455 (1994).

[10] M.I.H. Bhuiyan, M.O. Ahmad and M.N.S. Swamy, "Spatially adaptive thresholding in wavelet domain for speckling of ultrasound images", IET Image Processing, 3(3): 147-162 (2009).

[11] A. Pizurica, W. Philips and I. Lemahieu, "A versatile wavelet domain noise filtration technique for medical imaging", IEEE Transactions on Medical Imaging, 22(3): 323-331 (2003).

[12] D. Joachim, C.H. Per and H.J. Soren, "Algorithms and software for total variation image reconstruction via first-order methods", Numerical Algorithms, 53(1):67-92 (2010).

[13] L. Sendur and I. Selesnick, "Bivariate shrinkage functions for wavelet-based denoising exploiting interscale dependency”, IEEE Transactions on Signal Processing, 50(11): 2744-2756 (2002).

[14] I. Selesnick, R. Baraniuk and N. Kingsbury, "The dual-tree complex wavelet transform", IEEE Signal Processing Magazine, 22(6): 123-151 (2005). 